\title{
DYNAMIC AND STATIC WETTABILITY OF ADVANCED MATERIALS USED IN AERONAUTICAL APPLICATIONS
}

\author{
OMID GOHARDANI \& DAVID W. HAMMOND \\ Department of Power and Propulsion, Cranfield University, Bedford, UK.
}

\begin{abstract}
The search for icephobic external surface materials in aircraft icing applications has been ongoing since the early days of aviation. Given the recognized superlative properties of carbon nanotubes (CNTs) across many different disciplines, the implementation of CNTs in polymer matrix composites has sparked a great interest in their mechanical/electrical properties and wetting character, in addition to their suitability for aircraft icing applications. Within this framework, a new developed methodology capable of determining the nature of wettability, consequent to CNT implementation is desired. Thus, this article presents a novel methodology - henceforth referred to as the dynamic and static wettability scheme for advanced materials - which examines the wettability of materials reinforced with CNTs, for potential utilization within the aerospace industry. The described methodology herein can be employed in order to numerically examine empirically acquired results with an extended possibility to include alternative materials outside the scope of the considered ones. Results are shown for a decision matrix that discriminates between hydrophobic and hydrophilic surfaces based on their static and dynamic wettability properties. Moreover, idealized wetting character representations of the different considered aerospace materials are presented.

Keywords: aerospace materials, aircraft icing, carbon nanotube wettability, corona splashing measurement tool, dynamic and static wettability scheme for advanced materials, fluid structures, hydrophilicity, hydrophobicity, liquid water concentration.
\end{abstract}

\section{INTRODUCTION}

The usage of polymeric matrix composites has become more frequent in a number of different industries including electronics, the automotive and the aerospace industry [1]. The aerospace sector in particular has embraced the usage of polymeric matrix composites in civil and military aircraft, often with weight consideration in mind. Due to the superior mechanical and physical properties that have been observed in carbon nanotubes (CNTs), a natural consideration is whether CNTs can be utilized as reinforcing agents to further enhance the mechanical and physical properties of polymeric matrix composites. For this reason, researchers have conducted various research efforts in order to examine the influence of CNTs on the mechanical properties of the aforementioned materials [2,24]. A factor of great importance in aerospace applications due to high speed flight of, for instance, Earth entry vehicles and aircraft, is erosion [3]. In particular, Gohardani et al. [4], examined the erosion resilience, mechanical properties and acoustic impedance of a selection of polymer matrix composites reinforced with CNTs at $0.5 \%$ wt. for potential use in aeronautics. In this study, some disparities were observed between the different properties of the materials, but nominally similar erosion resilience values for the considered materials were encountered despite the presence of CNTs. The reasoning behind this observation was attributed to the dispersion process of CNTs in the resin. The influence of CNTs on the hydrophobic and hydrophilic nature of a set of specimens was further considered by Gohardani and Hammond [5] based on empirical results. Following the introduction of novel propulsion cycles such as distributed technology $[6,7]$ and their perspective applications [8], the present study describes a proposed methodology by Gohardani and Hammond [9] and exhibits numerical representations of the wettability of a number of CNT reinforced composites with potential usage within aeronautics. 


\section{EXPERIMENTAL SETUP}

The dynamic wettability experiments were carried out at the vertical droplet tunnel located at Cranfield University, United Kingdom. The flow was powered by a centrifugal backward curved suction fan, capable of producing flow rates, $\dot{m}_{\text {Fan }} \in[30,100] \mathrm{kg} \cdot \mathrm{s}^{-1}$. The temperature range $T \in[-30,+30]{ }^{\circ} \mathrm{C}$, was obtained by the air and refrigeration plant with a capacity of $400 \mathrm{~kW}$, capable of extracting the heat from the air [10]. Wetting of a target specimen was attained by a droplet generator ejecting mono dispersed droplets at a frequency of $f_{d} \approx 12 \mathrm{kHz}$, positioned at the entrance section of the droplet tunnel. Figure 1, shows a rendering of the experimental setup for the dynamic wettability experiments.

Two different illumination techniques were utilized in order to visualize the droplets and the wetting characteristics of the surface. The LED captured splashing features apparent in the volume of space along the width of each specimen, while the $2 \mathrm{~mm}$ laser sheet was employed in order to establish the local liquid water concentration. The image acquisition was actualized by employing a Sony XCD-SX90 camera operated at 30 FPS. The experimental images were acquired upon utilizing a graphical user interface (GUI) on a personal computer.

\subsection{Material substrates}

A total number of 10 different materials referred to hereafter with a specimen number $S_{n}$ were utilized in this study, in two different prescribed conditions; as supplied and in an eroded condition, with $n=\{1,2, \ldots, 10\}$. The majority of these specimens were established epoxy resins commonly used within the aerospace industry with addition of CNTs as a reinforcing agent. Specimens $S_{1}$ and $S_{2}$ were both combinations of Araldite ${ }^{\circledR}$ LY564/Aradur ${ }^{\circledR} 2954$ with the exception that specimen $S_{2}$ also featured multi-walled CNTs (MWCNT) at $0.5 \%$ wt. Graphistrength ${ }^{\circledR}$ C100. Specimens $S_{3}, S_{4}, S_{5}$ and $S_{6}$ were Araldite ${ }^{\circledR}$ MY0510/Aradur ${ }^{\circledR}$ 976-1

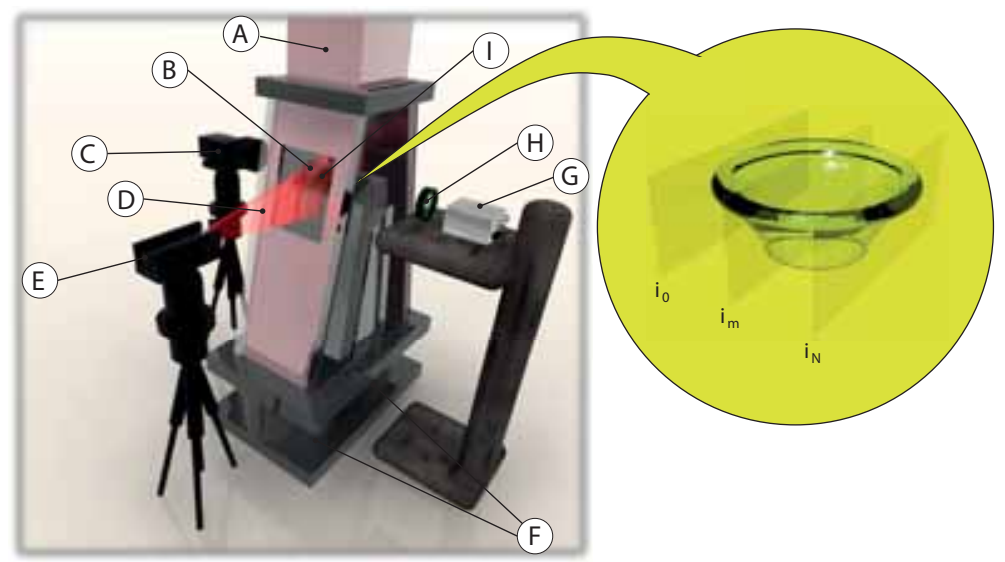

Figure 1: The experimental setup for the dynamic wettability experiments with notations: (A) Vertical droplet tunnel, (B) droplet cloud, (C) charge-coupled device camera, (D) laser sheet, (E) laser, (F) control valves, (G) light-emitting diode (LED) strobing device, $(\mathrm{H})$ collimating lens, and (I) specimen target. The right hand side image depicts a schematic corona structure with virtual planes $i_{0}, \ldots, i_{N}$, with $i_{m}$ showing the mid-plane of the corona. 
combinations, with $S_{4}$ and $S_{6}$ having $0.5 \%$ wt. MWCNT as a reinforcement. Specimen $\mathrm{S}_{7}$ was an Araldite ${ }^{\circledR}$ DBF/Aradur ${ }^{\circledR}$ HY956EN base resin with $10 \%$ wt. aluminum nitride nanoparticles, and specimen $S_{8}$ a gelcoat consisting of a SW404/XB5173 combination. Adopting this nomenclature classified specimens $S_{2}, S_{4}$ and $S_{6}$ as reinforced with CNTs. The materials were examined both in their pristine state and upon a wet blasting process which resulted in an averaged degraded surface finish $R_{a}^{e} \cong 4 \mu \mathrm{m}$, implying a higher surface roughness in relation to the averaged surface roughness value in the pristine state.

\section{THE DSWSAM METHODOLOGY}

The Dynamic and Static Wettability Scheme for Advanced Materials, henceforth referred to as (DSWSAM) describes the adopted methodology that has been utilized for image analysis of droplet splashing on the experimental specimens. The utilized approach comprises of determining the type of specimen based on its wetting characteristics, by employing the flowchart in Fig. 2.

Initially, a free stream velocity for the experiments was chosen on the basis of proximity to a reasonable flight speed within the range supported by the experimental facility. This choice further inhibited complexities of possible shock waves and aerodynamic shattering of the droplets. Upon choice of the velocity, the liquid water concentration was obtained based on a numerical scheme that identified the droplet cloud distribution from experimental photographs and provided an averaged histogram of the cloud spatial distribution on the target. This was attained upon input of the resulting photographic information into two different developed GUI tools in MATLAB ${ }^{\circledR}$, the liquid water content module (LWCM) and the corona splashing measurement tool (CSMT). The dynamic wetting photographs ascribed in the preand post-erosion state, were utilized as inputs to the DSWSAM methodology. For all analyzed images, initially the location of the specimen surface was determined. For certain images the actual location of the surface was obscured by the presence of a partial or continuous water film. Three different categories were therefore identified based on the waviness of the water film on the surface, as shown in Fig. 3.

The three different categories identified for the experimental images, were surfaces of hydrophobic, hydrophilic and hydrophobic/hydrophilic nature. The surface was classified as hydrophobic, if the specimen surface was partially visible and only obstructed by discrete
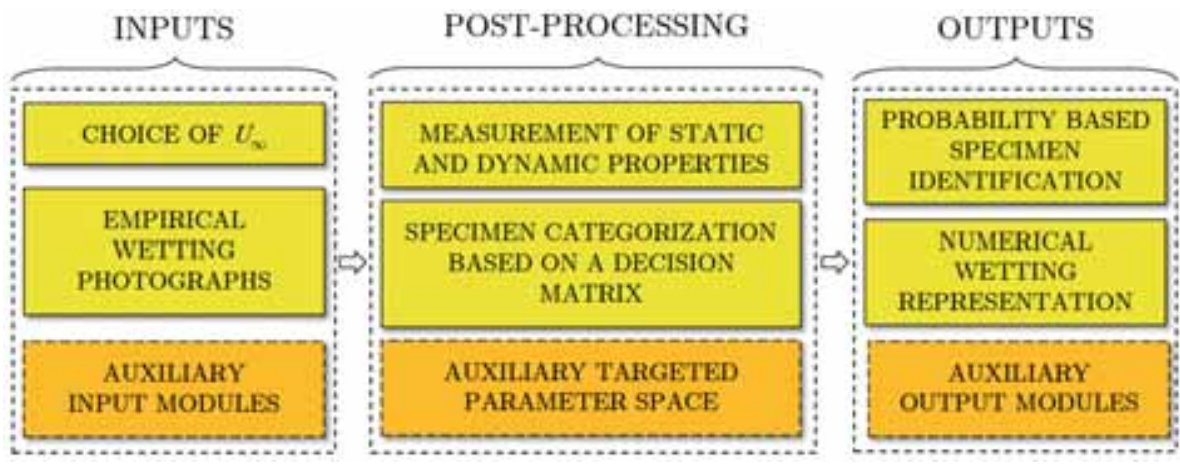

Figure 2: Flowchart of inputs, post-processing and data outputs of the DSWSAM methodology based on empirical results. Auxiliary modules can additionally target other parameter spaces and provide supplementary outputs, than those described in this study. 


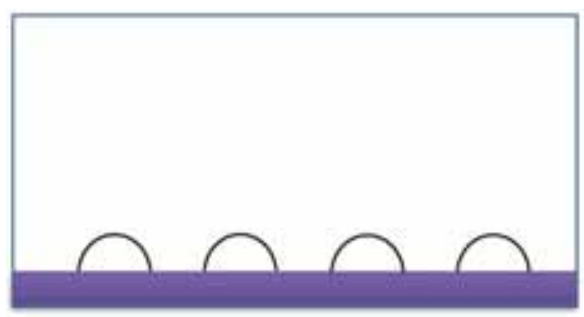

(a)

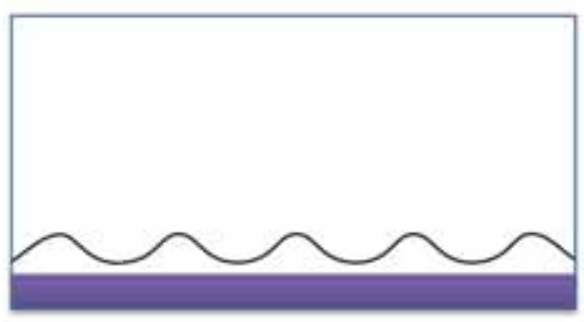

(c)

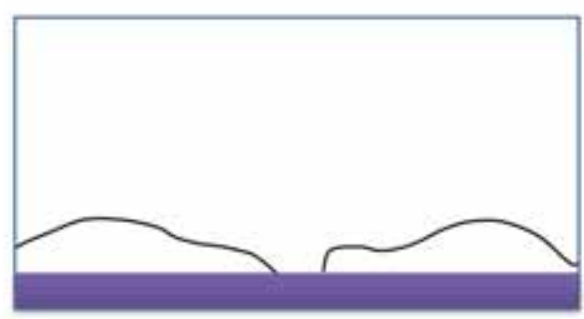

(e)

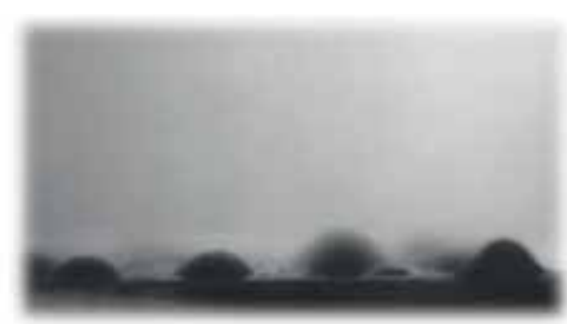

(b)

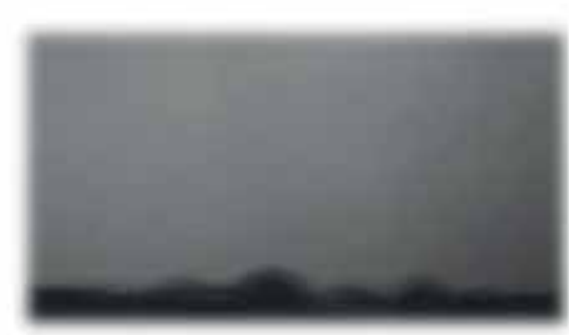

(d)

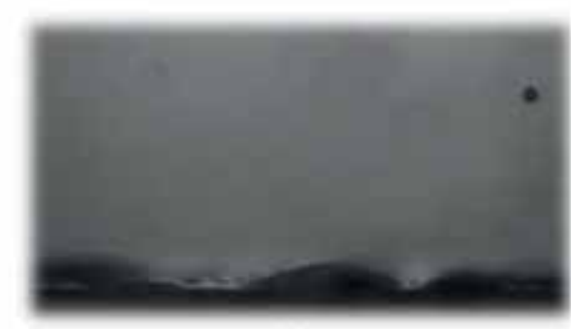

(f)

Figure 3: Idealized hydrophobic and hydrophilic wetting structures on different specimen surfaces with corresponding representative experimental photographs. Examples of hydrophobic surfaces are shown in (a) and (b). Figures (c) and (d) show a hydrophilic surface. Figures (e) and (f) finally represent a hydrophobic/hydrophilic surface.

ligaments of water, as shown in Figs. 3 (a) and (b). If a continuous water film layer was present on the specimen surface, it was categorized as a hydrophilic surface, as shown in Figs. 3 (c) and (d). For instances where an intermediate water film layer with partial characteristics of a continuous water film was identified, the specimen was either classified as hydrophilic or hydrophobic, visualized in Figs. 3 (e) and (f). For hydrophobic surfaces based on Figs. 3 (a) and (b) and the corresponding description, the number of fluid ligaments along the surface of the specimen were counted. A larger number of ligaments in this respect represented a more hydrophobic surface. For a hydrophilic surface, Figs. 3 (c) and (d), and a surface where the hydrophobic or hydrophilic surface character was difficult to categorize, as shown in Figs. 3 (e) and (f), the averaged wave lengths of the structures were determined. As the interaction of droplets on the specimen surface with and without an existing wetting character during the experiments largely resulted in formation of coronas, the structure of a water splash corona 


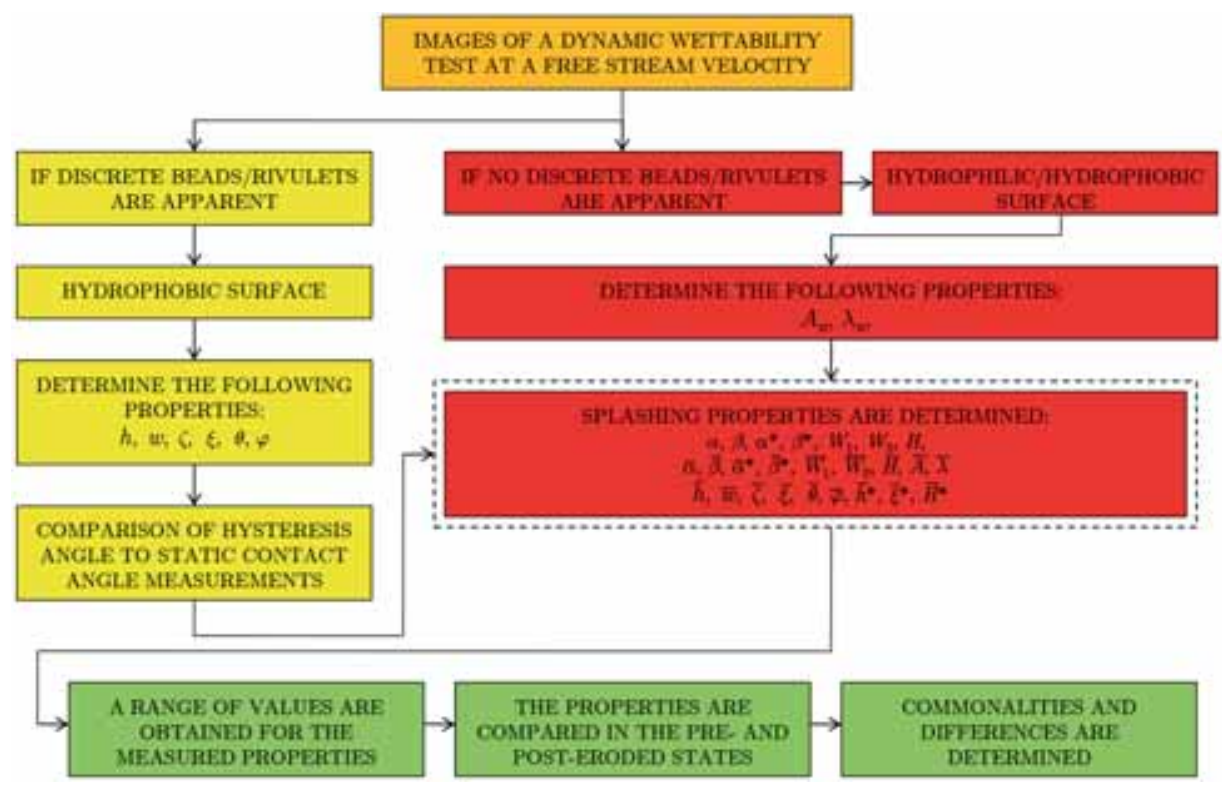

Figure 4: Methodology for the dynamic wettability test analysis.

was the only feature that could be compared across all considered specimens. For comparison purposes, the geometrical attributes of a corona structure consisting of the two angles that the jets subtended with the specimen surface, the width of the corona at base and top respectively, as well as the height of the corona were of interest, as these features could serve as a probe to determine the surface character and discriminate between the different surfaces.

The methodology depicted in Fig. 4, makes use of three important aspects of the wetting based on $(i)$ the wetting conditions, (ii) the wetting character of the surface and (iii) the resulting fluid structures upon water droplet impact. The wetting conditions were largely determined by the local liquid water concentration at which the experiments were carried out. The wetting character of the surface was described by either beads, rivulets or partial and complete water film layers. The resulting fluid structures in this study have been the formation of coronas upon splashing on the surface of the specimen. For each of the aforementioned modules, a tool was developed that enabled a simplified assessment of the wetting characteristics of the considered specimen. In particular, the wetting condition was addressed by the LWCM. The wetting character of the surface was determined by usage of an idealized fluid structure categorization and a mathematical description of a representative water film layer. The resulting fluid structures ensuing upon water droplet impacts were further analyzed by employing the CSMT.

\subsection{Static wettability}

Contact angle measurement were carried out on the candidate materials, in order to obtain the advancing and receding angle for each specimen. This method conveyed results about the surface energy using Young's equation [11]

$$
\gamma \mathrm{SL}-\gamma \mathrm{SL}-\cos (\varphi)=0
$$


where $\gamma$ is the energy and the subscripts SG and SL denote solid-gas and solid-liquid respectively. The drop was modified by either dispensing or retracting its volume, resulting in an advancing angle $\theta_{A}$ or a receding angle $\theta_{R}$, measured by a protractor. This method allowed for an assessment of the homogeneity of the specimen, as droplets could be deposited on various locations on the specimen surface. In this study an averaged value of five measurements placed on different locations on the surface is presented. Hysteresis $\psi$, can in this context be defined as $\psi=\theta_{A}-\theta_{R}$. The equilibrium Young contact angle $\theta_{C}$ can further be expressed in terms of the advancing and receding angles [12] as

$$
\theta_{C}=\arccos \left\{\frac{\Gamma_{A} \cos \left(\theta_{A}\right)+\Gamma_{R} \cos \left(\theta_{R}\right)}{\Gamma_{A}+\Gamma_{R}}\right\}
$$

where

$$
\Gamma_{A, R}=\left\{\frac{\sin ^{3}\left(\theta_{A, R}\right)}{2-3 \cos \left(\theta_{A, R}\right)+\cos ^{3}\left(\theta_{A, R}\right)}\right\}^{1 / 3}
$$

The static wetting of the specimens was assessed by employing contact angle measurements on both pristine and eroded specimen surfaces. The experimental setup for the contact angle measurements, is shown in Fig. 5. The specimen was positioned on a table with a vertical adjustment capability and illuminated by an LED with a diffuser, which provided a uniform

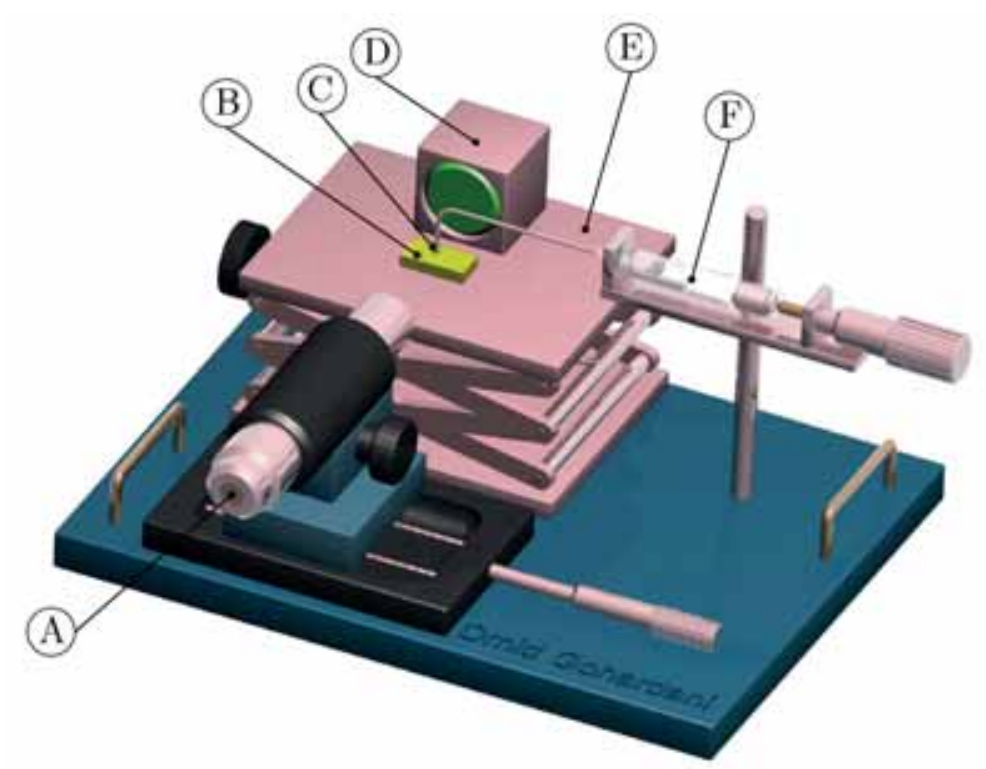

Figure 5: The experimental setup for the contact angle measurements, with notations: (A) Eye piece fitted with a protractor, (B) specimen, (C) generated droplet, (D) LED with diffuser, (E) vertical adjustable table, and (F) syringe and spring device fitted with a micrometer. 
illumination of the droplet. The 5 cc syringe with a glass luer slip tip, fitted with a 6 " needle and $90^{\circ}$ blunt end, produced the $2.04 \mathrm{~mm}$ drop of deionized water. The volume of fluid utilized for these measurements ranged between 10-90 $\mu$ l. The uncertainty of the measurements was mainly related to the manual goniometry readings of the back-lit droplet's advancing and receding angles. Since imperfections in surface uniformity and cleanness could be present at the time of the readings, an error of $\sim 5^{\circ}$ was expected for the contact angle measurements. The setup utilized for measurement of the advancing and receding contact angles on $S_{1}-S_{10}$ is shown in Fig. 5.

\subsection{Wetting condition}

\subsubsection{Liquid water concentration module}

In order to obtain the local liquid water content $(L W C)$, experimental photographs from 10 experiments were considered. Each experiment consisted of 347 images acquired under a sampling time $\tau \approx 10$ seconds at 30 FPS. The LWCM developed in MATLAB ${ }^{\circledR}$ processed each experimental image separately. On the acquired photographs the droplets were visible as white streaks against a black background.

Upon setting a normalized contrast level for all images, the droplet tracking scheme was employed in order to find the centroid positions of each droplet, while the off-plane droplets were neglected in accordance with Fig. 6.

The position of each centroid on the image can be denoted as $\{C(x, y)\}$ i. For each image, the total number of centroids was hence identified, resulting in the total number of centroid positions for the droplets within each frame, described by

$$
C_{\text {Total }}=\left[\sum_{i=1}^{N}\{C(x, y)\}_{i}\right]_{j}
$$

In order to produce a histogram of the number of droplets within each frame, a nondimensional length $L^{*}=x / L$ was introduced with $L^{*} \in[0,1]$. The non-dimensional length

(a)

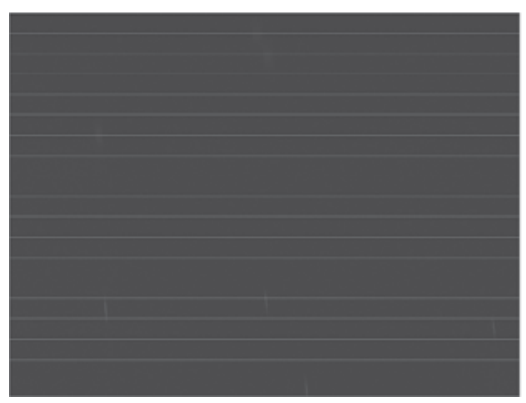

(b)

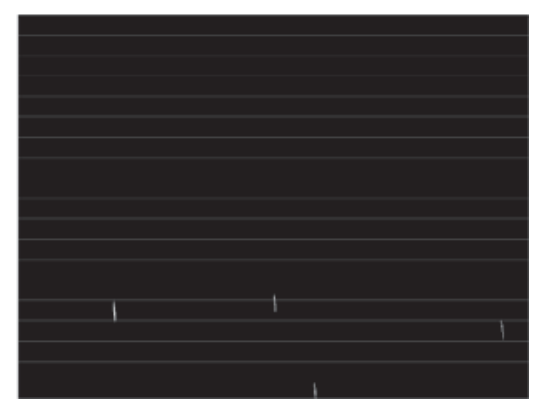

Figure 6: Droplet counting by the LWCM. The original acquired experimental photograph is shown to the left with the droplets extremely intricate to distinguish as shown in (a). The modified binary image to the right displays a few droplets, shown as white streaks in (b). 
along the specimen was further divided into 10 equally sized bins. The allocation of each centroid position $i$, to each bin $k$, was actualized by

$$
\frac{n L^{*}}{10} \leqq C\left(L^{*}\right)_{i}<\frac{(n+1) L^{*}}{10}
$$

with $n=0,1, \ldots, 9, i=1,2, \ldots, N$ and $k=1,2, \ldots, 10$.

For each centroid position fulfilling eqn (5), an increment was added to the total number of droplets within each bin $m_{k}$. The total number of droplets for a given frame was hence given by

$$
N_{j}=\sum_{k=1}^{N} m_{k}
$$

The averaged number of droplets within a sequence of images, in a given zone, were further given by

$$
\bar{R}_{j}=\frac{1}{n}\left\{\sum_{i=1}^{n} D_{i}\right\}_{j}
$$

where $i=\{1,2, \ldots, n\}, j=\{1,2, \ldots, 10\}$ and $n$ is the number of frames equal to 347 . With known number of droplets, a similar approach for estimation of the local Liquid Water Content $(L W C)$ as Ide [13] was employed. For a constant droplet diameter, $D$ and total sample area for the bins $\tilde{A}$, the $L W C$ can be expressed as

$$
L W C \cong \frac{\rho \pi D^{3}}{6 \tilde{A} U_{\infty} t} \sum_{i} R_{j}
$$

where $\rho$ is the water density, $t$ is the sampling time, $U_{\infty}$ is the free stream velocity, and the sum refers to the total number of droplets.

\subsection{Wetting character of the surface}

In order to adopt a nomenclature that could easily be measured empirically and identified analytically, a number of different structures describing possible wetting features of the surfaces were defined, as shown in Fig. 7.

Four fluid structure classifications, Categories I-IV were identified within the population, to be present on the specimen surface, upon impact of the droplet stream onto the surface, within the field of view of the camera. The parameters shown in Fig. 7 refer to the measured parameters and the output column to results produced by this module. Categories I and II, represent idealized bead and rivulet structures, respectively. As fluid structures of each presented category in Fig. 7, feature different geometrical sizes, a representative fluid structure for each category was determined upon averaging over a large population of images, with the considered structures.

The distinction between a bead (Category I) and a rivulet (Category II) was largely determined by $\xi \gg w$, for a rivulet, while $h \sim \zeta$. The distinction criterion employed herein was that a structure was categorized as a rivulet if $\xi>\ell / 2$, where $\ell$ is the length of the specimen within the field of view. This criterion, clearly distinguished between beads and rivulets as a more moderate length restriction on $\xi$ would make the distinction more difficult. Due to the larger spatial extent of a rivulet, it can be stated that the number of beads in general was greater than 


\begin{tabular}{|c|c|c|c|}
\hline CATEGORY & GEOMETRY & PARAMETERS & OUTPUTS \\
\hline $\begin{array}{l}\text { BEADS } \\
\text { (I) }\end{array}$ & $\stackrel{\leftrightarrow}{\longleftrightarrow}$ & $w, h, \theta$ & $\begin{array}{c}\bar{h} / \bar{w}, \bar{w} / \bar{h} \\
\bar{\theta}, n_{b}, n_{b, c}\end{array}$ \\
\hline $\begin{array}{l}\text { RIVULETS } \\
\text { (II) }\end{array}$ & & $\xi, \zeta, \varphi$ & $\begin{array}{c}\bar{\xi}, \bar{\zeta}, \bar{\xi} / \bar{\zeta}, \bar{\zeta} / \bar{\xi} \\
\bar{\varphi}, n_{r}, n_{r, c}\end{array}$ \\
\hline $\begin{array}{l}\text { WATER } \\
\text { FILM } \\
\text { (III) }\end{array}$ & & $\begin{array}{c}A_{1}, \ldots, A_{n} \\
\lambda_{1}, \ldots, \lambda_{n}\end{array}$ & $\begin{array}{l}\bar{A}, \bar{\lambda} \\
f_{w f, e}\end{array}$ \\
\hline $\begin{array}{c}\text { CORONA } \\
\text { (IV) }\end{array}$ & $W_{2}$ & $\begin{array}{c}\alpha, \beta, \alpha^{*}, \beta^{*} \\
W_{1}, W_{2}, H\end{array}$ & $\begin{array}{c}h^{*}, \xi^{*}, H^{*} \\
W_{1}, W_{2}, H \\
\bar{\alpha} / \bar{\beta}, \bar{\beta} / \bar{\alpha} \\
\bar{\alpha}^{*} / \bar{\beta}^{*} \\
\bar{\beta}^{*} / \bar{\alpha}^{*} \\
E_{C} / E_{F}\end{array}$ \\
\hline
\end{tabular}

Figure 7: Fluid structure categories with corresponding measured parameters and outputs upon employing the DSWSAM methodology.

the number of rivulets, $n_{b}>n_{r}$. A large population of beads on the surface $n_{b} \gg 1$, further inferred that the surface was hydrophobic.

A continuous water film (Category III) was present in the case where $\xi \rightarrow \infty$, with the amplitude of each wave $A_{i}>0$. A partial water film was further apparent when $A_{i}>0$ was followed by $A_{i+1} \rightarrow 0$ and $A_{i+2}>0$, along the entire surface of the specimen, in a continuous manner. Hence, it could readily be established that Category II differed from Category III, as it had a more discrete character.

The ensuing fluid structure following the impact of an incoming droplet of size d, was a corona structure, shown in Category IV. The employed nomenclature hence determined the corona structure parameters regardless of the initial formed surface fluid classification (Categories I-III). A set of parameters that addressed a comparison between the incoming and splashing structure were hence, $h^{*} \equiv H /(\bar{h}+d), \zeta^{*} \equiv H /(\bar{\zeta}+d)$ and $A^{*} \equiv H /(d+\bar{A})$. The reasoning behind analyzing the geometrical parameters of the corona structures across all considered specimens was to provide a metric for wettability of each material. In particular this method was useful, upon analyzing the wetting character of the pre- and post eroded specimens, as it provided an insight into the influence of erosion on the wetting character of each specimen.

The defined structures in Fig. 7, were idealized for simplicity and described merely by the shown geometrical properties. Although shape deviations from the idealized structures existed in comparison to the encountered structures during the experimental runs, the defined geometrical structures at large, adequately captured the behavior of the fluid characters on the surface and allowed for simple measurements of the fluid features by utilizing a personal computer. 
In order to analyze the experimental photographs, a baseline for the location of the top surface of the specimen was established by utilizing the ImageJ software. All categories except Category IV were thereafter discretized by positioning of nodes $n_{i}=\{1,2, \ldots, N\}$, using the multi-point tool in ImageJ. The intermediate distance between the points, $\Omega_{i}=\left|x_{\mathrm{i}+1}-x_{\mathrm{i}}\right|$ hence represented different length scales where $\Omega_{i}=\lambda_{i}$ denoted the wavelength of the water film, $\Omega_{i}=\omega_{i}$ width of a bead and $\Omega_{i}=\xi_{i}$ width of a rivulet, depending on the analyzed structures. The height of each structure $\Xi_{i}=\left|y_{\text {apex }}-y_{i}\right|$ was defined as the distance between the base and the apex of the structure, with $\Xi_{i}=A_{i}$ denoting the amplitude of the water film, $\Xi_{i}=h_{i}$ representing the height of a bead and $\Xi_{i}=\zeta_{i}$ identifying the height of a rivulet. A surface covered with beads could in an idealized representation consist of $n$ number of beads, each having a width $\omega_{n}$ and height $h_{n}$ placed adjacent to each other, described by

$$
B_{n}(x)=\frac{h_{n}\left(-4 x^{2}+8 x H_{n}-4 H_{n}^{2}+w_{n}^{2}\right)^{\frac{1}{2}}}{w_{n}}
$$

where the horizontal distance of each bead's apex position from the origin $H_{n}$ was defined as

$$
H_{n}(x) \equiv \begin{cases}\frac{w_{1}}{2} & , n=1 \\ w_{1}+\frac{w_{2}}{2} & , n=2 \\ H_{n-1}+w_{n-1}+w_{n} & , n>2\end{cases}
$$

A representative bead structure could further be expressed as

$$
\bar{B}(x)=\frac{\bar{h}\left(-4 x^{2}+\bar{w}\right)^{2}}{\bar{w}}
$$

with $\bar{h}=\sum_{n=1}^{N} h_{i} / n$ and $\bar{w}=\sum_{n=1}^{N} w_{i} / n$ determined from the experiments. The idealized water film layer could similarly be expressed by

$$
\bar{W}(x)=\bar{A} \sin (\omega x)+\tilde{B}
$$

where $\tilde{B}$ is a constant, $\omega=2 \pi /|\bar{\lambda}|$ and the parameters $\bar{A}=\sum_{n=1}^{N} A_{i} / n$ and $\bar{\lambda}=\sum_{n=1}^{N} \lambda_{i} / n$ were determined upon discretization using the empirical photographs. The water film layer was then confined between the function $\bar{W}(x)$ and $y=0$, for $x \in[0, \infty)$.

Upon visual representation of the results, it is often useful to express the parameters in non-dimensional form in order to facilitate the comparison between different surfaces regardless of the length of the specimen. The used notation herein is based on the total length of the specimen in the field of view $\ell$, upon empirical image acquisition. For a bead, the nondimensional height and width were given by $h^{*}=\bar{h} / \ell$ and $w^{*}=\bar{w} / \ell$. The number of beads was further enumerated by $n_{s t}$ and the number of spacings between the beads given by $n_{s p}$. These notations hence implied that $n_{s t}>n_{s p}$.

The event where $n_{s t} \gg n_{s p}$ was evident, upon a cluster of beads being encountered on the surface and placed adjacent to each other. Similarly for a rivulet, the height and width were denoted by $\zeta^{*}=\bar{\zeta} / \ell$ and $\xi^{*}=\bar{\xi} / \ell$. For rivulets, the number of spacings were not considered, allowing the plurality of rivulets to be represented by a single rivulet with the mean value of height and width established by the considered rivulets. Lastly for a water film $A^{*}=\bar{A} / \ell$, denoted the non-dimensional amplitude and $\lambda^{*}=\bar{\lambda} / \ell$, the non-dimensional wavelength of the water film. In the idealized representations of the surface structures, shown in Figs. 11 and 12 , only the number of bead structures $n_{s t}$ was considered. 


\subsection{Resulting fluid structures upon impact}

A collision of a water droplet with a specimen surface may result in different scenarios in which bouncing, spreading and splashing may occur [14]. For splashing structures, the nature that follows is largely dependent upon the depth of the water layer, the incident angle, droplet speed and the wettability of the target. For the chosen droplet size $D=400 \mu \mathrm{m}$, it was empirically observed that this size resulted in a large number of impacts and splashing corona structures, due to the local $L W C$ and was capable of exposing the target area to adequate wetting. This level of wetting would hence expose the nature of hydrophobic specimens to beads and rivulets, and the nature of hydrophilic specimens to the formation of continuous layers of water films. The formation of corona structures in the aftermath of the water droplet collision with the surface, hence enabled a complete comparison of the geometrical parameters of the corona structure regardless of the wetting character of the surface, across the entire scope of the given specimens.

\subsubsection{The CSMT}

A caveat of analyzing images with respect to spatial measurements and angles, ensues when the population of images exceeds 100 . As the number of acquired empirical images in this study exceeded 16,000 , the development of a measuring tool was a necessity for numerous reasons. In particular, consistency during the measurements and robustness in obtaining the results across the entire population was desired. For this reason, the CSMT was developed in MATLAB ${ }^{\circledR}$. Figure 8 shows the CSMT positioned on top of an actual empirical image of a corona structure.

The CSMT is based upon positioning of 4 vertices on the empirical image by the user, on top of the location of the corona structure. The resulting geometry consists of vectors $\mathbf{v}=\left\{\mathbf{v}_{1}\right.$, $\mathbf{v}_{2}, \ldots, \mathbf{v}_{4}$ \} placed counter-clockwise with the initial position at the left upper corner of the corona structure. The angle a subtended by the structure is therefore described by

$$
a=\arccos \left(\frac{\mathbf{v}_{1} \cdot \mathbf{v}_{2}}{\left\|\mathbf{v}_{1}\right\| \cdot\left\|\mathbf{v}_{2}\right\|}\right)
$$
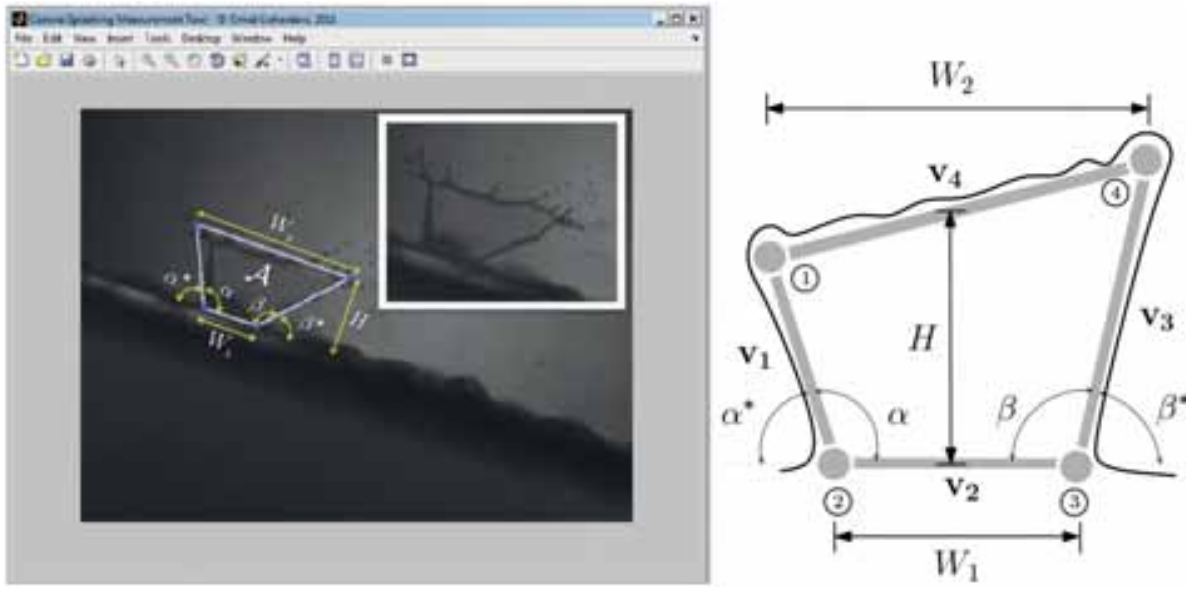

Figure 8: The Corona Splashing Measurement Tool (CSMT), developed in MATLAB ${ }^{\circledR}$. 
Similarly the angle $\beta$ is given by

$$
\beta=\arccos \left(\frac{\mathbf{v}_{2} \cdot \mathbf{v}_{3}}{\left(\left\|\mathbf{v}_{2}\right\| \cdot\left\|\mathbf{v}_{3}\right\|\right)}\right)
$$

In addition, the angles fulfill the requirement of $\alpha+\alpha^{*}=180^{\circ}$ and $\beta+\beta^{*}=180^{\circ}$. The height of the corona $H$, is with the given nomenclature described by

$$
H=\frac{\eta}{2\left(\left\|\mathbf{v}_{4}\right\|-\left\|\mathbf{v}_{2}\right\|\right)}
$$

where

$$
\eta=4 \sqrt{\left(s-\left\|\mathbf{v}_{2}\right\|\right)\left(s-\left\|\mathbf{v}_{4}\right\|\right)\left(s-\left\|\mathbf{v}_{4}\right\|-\left\|\mathbf{v}_{3}\right\|\right)\left(s-\left\|\mathbf{v}_{4}\right\|-\left\|\mathbf{v}_{1}\right\|\right)}
$$

and $s$ is given by

$$
s=\frac{\left\|\mathbf{v}_{1}\right\|+\left\|\mathbf{v}_{2}\right\|+\left\|\mathbf{v}_{3}\right\|+\left\|\mathbf{v}_{4}\right\|}{2}
$$

The two-dimensional area of the corona structure is hence given by

$$
A \approx \frac{H \cdot\left(\left\|\mathbf{v}_{2}\right\|+\left\|\mathbf{v}_{4}\right\|\right)}{2}
$$

In order to estimate the amount of variability in pixel intensity within the region of interest subtended by the 4 vertices of the corona structure, the entropy within the region of the experimental photographs was computed. The entropy for a grayscale image in MATLAB ${ }^{\circledR}$, is defined as a measure of the randomness associated with the texture of the image [15], and is computed according to

$$
\tilde{E}=-\sum p \cdot \log _{2}(p)
$$

where $p$ contains the count of the histogram. This approach is applied both to the entropy of the entire image resulting in the entropy of the frame, $E_{F}$ and confined only to the corona for which $E_{C}$ is computed. This parameter provides an insight into the uniformity of the pixel intensity within the corona structure, as recorded on the experimental photograph. Smooth textures hence result in lower and rough textures in higher entropy values. The CMST produces measurements of the corona structure given by the generic function

$$
\Phi=f\left(\alpha, \beta, \alpha^{*}, \beta^{*}, W_{1}, W_{2}, H, \mathcal{A}, E_{C}, E_{F}\right)
$$

The CSMT outputs the parameters given in eqn (20) for a given initial position of the vertices designated by $p_{i}\left(x_{i}, y_{i}\right)$ with $i=1 \ldots 4$, implying in an output from the generic function $\Phi_{p}$. If the position of any of the $i$ :th coordinates is altered resulting in $\mathrm{p}_{i}^{\prime}\left(\mathrm{x}_{i}^{\prime} ; \mathrm{y}_{i}^{\prime}\right)$ with $i=1 \ldots 4$, and $p \neq p^{\prime}$, the software is capable of producing a new generic function $\Phi_{p^{\prime}}$. The repositioning procedure can hence be carried out until the vertices of the CSMT are superimposed on top of the four edges of the corona structure on the empirical image. This results in a final generic function for the specific frame $\Phi_{F}$, with $F \in[0, N]$, where $N$ is a positive integer. Based on the measured parameters, a range of non-dimensional factors were obtained for the splashing 
structures. These properties were measured both in the pre- and post eroded states of the specimens, respectively and their commonalities and differences were determined. Four different combination of geometrical parameters were considered in order to establish the behavior of the corona structure. The initial one examined the skewness of the corona by determining the ratio $\alpha^{*} / \beta^{*}$. In this respect, three different cases could unfold according to:

$$
\frac{a^{*}}{\beta^{*}}= \begin{cases}\text { Right }- \text { skewed corona } & >1 \\ \text { Neutral corona } & =1 \\ \text { left }- \text { skewed corona } & <1\end{cases}
$$

The second parameter of interest was $\left(\bar{W}_{2}-\bar{W}_{1}\right) / \bar{W}_{1}$, which described the expansion of base width, to the top width of the corona, in percent. A value of $\left(\bar{W}_{2}-\bar{W}_{1}\right) / \bar{W}_{1}>1$, hence implied an expansion and $\left(\bar{W}_{2}-\bar{W}_{1}\right) / \bar{W}_{1}<1$, a reduction of width in comparison to the base width of the corona. The aspect ratio of the corona was furthermore given by $\left(\bar{W}_{1}+\bar{W}_{2}\right) / 2 \bar{H}$. A value of $\left(\bar{W}_{1}+\bar{W}_{2}\right) / 2 \bar{H}=1$, was indicative of a square shaped corona, and $\left(\bar{W}_{1}+\bar{W}_{2}\right) / 2 \bar{H}>1$, a rectangular shaped corona structure.

\subsection{The influence of the boundary layer}

In order to estimate the influence of the boundary layer on the incoming droplets, a simplified approach can be considered for estimation of the size of the boundary layer thickness. Under the assumption that the stagnation point is located sufficiently far away from the leading edge of the specimen, the flow over the specimen surface can be approximated as the flow over a flat plate with an incident angle $\alpha=0^{\circ}$. For a finite length of the plate $x$, the inertial force $\rho u(\partial u / \partial x) \sim \rho\left(U_{\infty}^{2} / x\right)$. The friction force per unit volume is however given by $d \tau / d y \sim\left(\mu U_{\infty}\right) / \delta^{2}$. Equating the inertial and friction forces yields $\delta(x) \sim\left\{(\mu x) /\left(\rho U_{\infty}\right)\right\}^{\frac{1}{2}}$. If the position of the boundary layer thickness is determined when $u=0.99 U_{\infty}$, the boundary layer thickness can be defined as [16]

$$
\delta_{99} \approx 5.0 \sqrt{\frac{v x}{U_{\infty}}}
$$

The reduction of volume flux due to the action of viscosity or the displacement thickness $\delta_{1}$ and other parameters such as the momentum thickness $\delta_{2}$ and the energy thickness $\delta_{3}$, are further given by eqns (23)-(25)

$$
\begin{gathered}
\delta_{1}=\int_{0}^{\infty}\left(1-\frac{u}{U_{\infty}}\right) d y \\
\delta_{2}=\int_{0}^{\infty} \frac{u}{U_{\infty}}\left(1-\frac{u}{U_{\infty}}\right) d y \\
\delta_{3}=\int_{0}^{\infty} \frac{u}{U_{\infty}}\left(1-\frac{u^{2}}{U_{\infty}^{2}}\right) d y
\end{gathered}
$$

Moreover, the wall shear stress is approximated by $\tau_{w}(x) \approx\left\{\left(\rho \mu U_{\infty}^{3}\right) / x\right\}^{\frac{1}{2}}$ and the skin-friction coefficient by $c_{f}(x) \approx 2 \tau_{w}(x) /\left(\rho U_{\infty}\right)^{2}$. The splashing phenomenon is often related to different non-dimensional parameters such as the Reynolds number based on the diameter $\operatorname{Re}_{d}=$ $\left(\rho U_{\infty} d\right) / \mu$, the Weber number $W e_{d}=\left(\rho U_{\infty}^{2} d\right) / \sigma$, the Ohnsorge number $O h=\sqrt{W e} / R e$, and the 
Capillary number $C a=W e / R e$, where $d$ is the droplet diameter, $\rho$ defines the density, $U_{\infty}$ is the free stream velocity, $\mu$ is the dynamic viscosity, and $\sigma$ denotes the surface tension. The spreading factor $\tilde{\xi}=d_{\max } / d$, can further be expressed as [17]

$$
\tilde{\xi}=\sqrt{\frac{W e+12}{3\left(1-\cos \left(\theta_{A}\right)+\frac{4 W e}{\sqrt{R e}}\right)}}
$$

\section{RESULTS AND DISCUSSION}

Table 1, shows the advancing, receding, equilibrium Young contact angle and their corresponding hysteresis.

The local liquid water concentration for a representative experiment with $U_{\infty} \approx 35 \mathrm{~m} \cdot \mathrm{s}^{-1}$ is shown in Fig. 9. Depictive empirical photographs for each specimen in their pristine and

Table 1: Surface roughness on the specimens in the pre- and post-erosion state given by $R_{a}^{p}$ and $R_{a}^{e}$ respectively. The percentage increase and decrease is given by $\Delta R_{a}=$ $\left(R_{a}^{p}-R_{a}^{e}\right) / R_{a}^{p}$. The advancing angle $\theta_{A}$, receding angle $\theta_{R}$, hysteresis $\psi$ and the equilibrium Young angle $\theta_{C}$ for the specimens are shown with the pristine value in the left and the eroded value to the right, in each column.

\begin{tabular}{rlllrrrrrrrr}
\hline$S_{n}$ & $R_{a}^{p}[\mu \mathrm{m}]$ & $R_{a}^{e}[\mu \mathrm{m}]$ & \multicolumn{1}{c}{$\Delta R_{a}$} & \multicolumn{2}{c}{$\theta_{A}\left[^{\circ}\right]$} & \multicolumn{2}{c}{$\theta_{R}\left[^{\circ}\right]$} & \multicolumn{2}{c}{$\psi\left[{ }^{\circ}\right]$} & \multicolumn{2}{c}{$\theta_{C}\left[^{\circ}\right]$} \\
\hline$S_{1}$ & 5 & 4.24 & -0.152 & 110 & 75 & 22 & 11 & 88 & 64 & 22 & 11 \\
$S_{2}$ & 0.74 & 4.4 & 4.946 & 93 & 90 & 33 & 6 & 60 & 84 & 35 & 6 \\
$S_{3}$ & 0.2 & 3.98 & 3.675 & 87 & 80 & 28 & 10 & 59 & 70 & 30 & 10 \\
$S_{4}$ & 0.8 & 3.74 & 4.946 & 80 & 90 & 17 & 10 & 63 & 80 & 18 & 10 \\
$S_{5}$ & 0.2 & 4.06 & 19.30 & 90 & 68 & 28 & 13 & 62 & 55 & 30 & 14 \\
$S_{6}$ & 1.2 & 3.92 & 2.267 & 93 & 70 & 32 & 8 & 61 & 62 & 33 & 8 \\
$S_{7}$ & 0.8 & 2.32 & 1.90 & 82 & 110 & 32 & 22 & 50 & 88 & 34 & 22 \\
$S_{8}$ & 0.6 & 2.98 & 3.967 & 117 & 90 & 60 & 8 & 57 & 82 & 63 & 8 \\
\hline
\end{tabular}

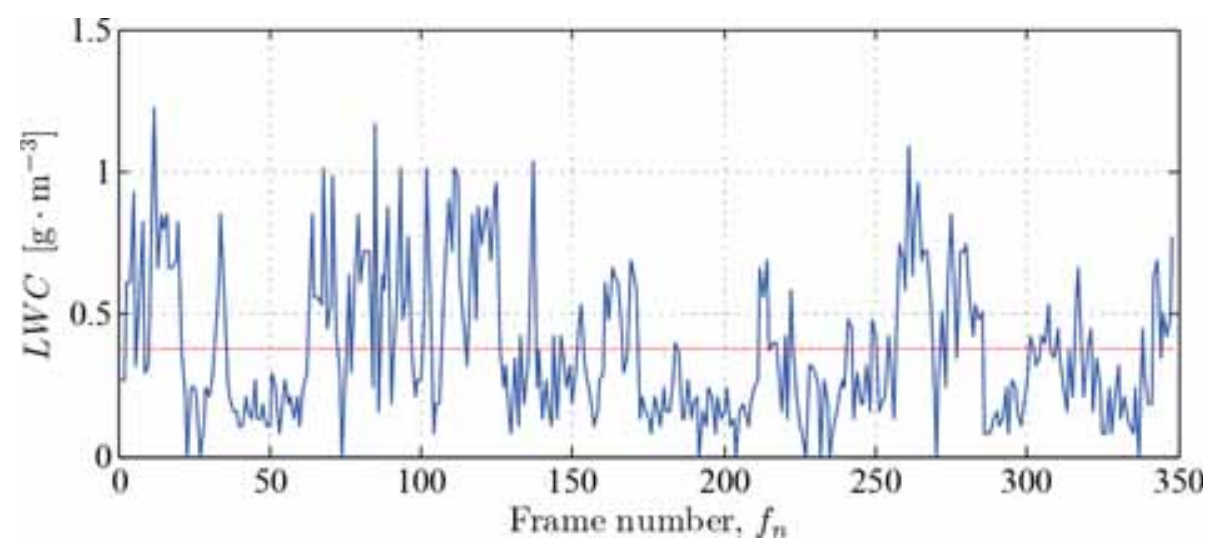

Figure 9: The local liquid water concentration versus the frame number for a $U_{\infty} \approx 35 \mathrm{~m} \cdot \mathrm{s}^{-1}$ experiment, showing $L W C \cong 0.38 \mathrm{~g} \cdot \mathrm{m}^{-3}$. 
eroded state respectively, are further shown in Fig. 10. A representative idealization of the wetting character of the surface with implementation of the non-dimensional parameters, is further presented in Figs. 11 and 12. From the figures it can be deduced that the idealized structures covered in the pristine state of the specimens consist of beads, rivulets and water films. Upon erosion of the surface a continuous water film is present on the surface. The aforementioned observations can qualitatively also be deduced upon visual inspection of the empirical photographs. The DSWSAM methodology is further able to discriminate between the ensuing water film character on the surface upon considering their different amplitudes and the wavelengths of the water films, depicted in (b), (d), (f) and (h) in Figs. 11 and 12.

In order to characterize the corona structure, the generic function $\Phi$ establishes the value of at least ten parameters that geometrically describe the corona structure. For a generic parameter $x$ such that $\Phi=f(\chi)$, the CSMT outputs the lower and upper bound of the parameter in the pristine and eroded settings, respectively, i.e. $\chi \in\left[\chi_{\min }^{p, e}, \chi_{\max }^{p, e}\right]$. Figure 13 shows the minimum and maximum range of the parameters $W_{1}, W_{2}, H$ and $E_{C} / \mathrm{E}_{F}$ for a free stream velocity of $U_{\infty} \approx 35 \mathrm{~m} \cdot \mathrm{s}^{-1}$. Similarly, Fig. 14 shows the minimum and maximum range of the angles of the corona, namely $\alpha, \beta$ and the complimentary angles $\alpha^{*}$ and $\beta^{*}$ for a free stream velocity $U_{\infty} \approx 35 \mathrm{~m} \cdot \mathrm{s}^{-1}$. The geometrical properties presented in Figs. 13 and 14 convey important behavior of the corona structure prior and after erosion of the surface. The first observation is that in large for all specimens, the base of the corona extends to a larger region. For the majority of the specimens this feature may arise from the fact that a more uniform surface finish is obtained allowing the water to spread more freely on the surface. It is also
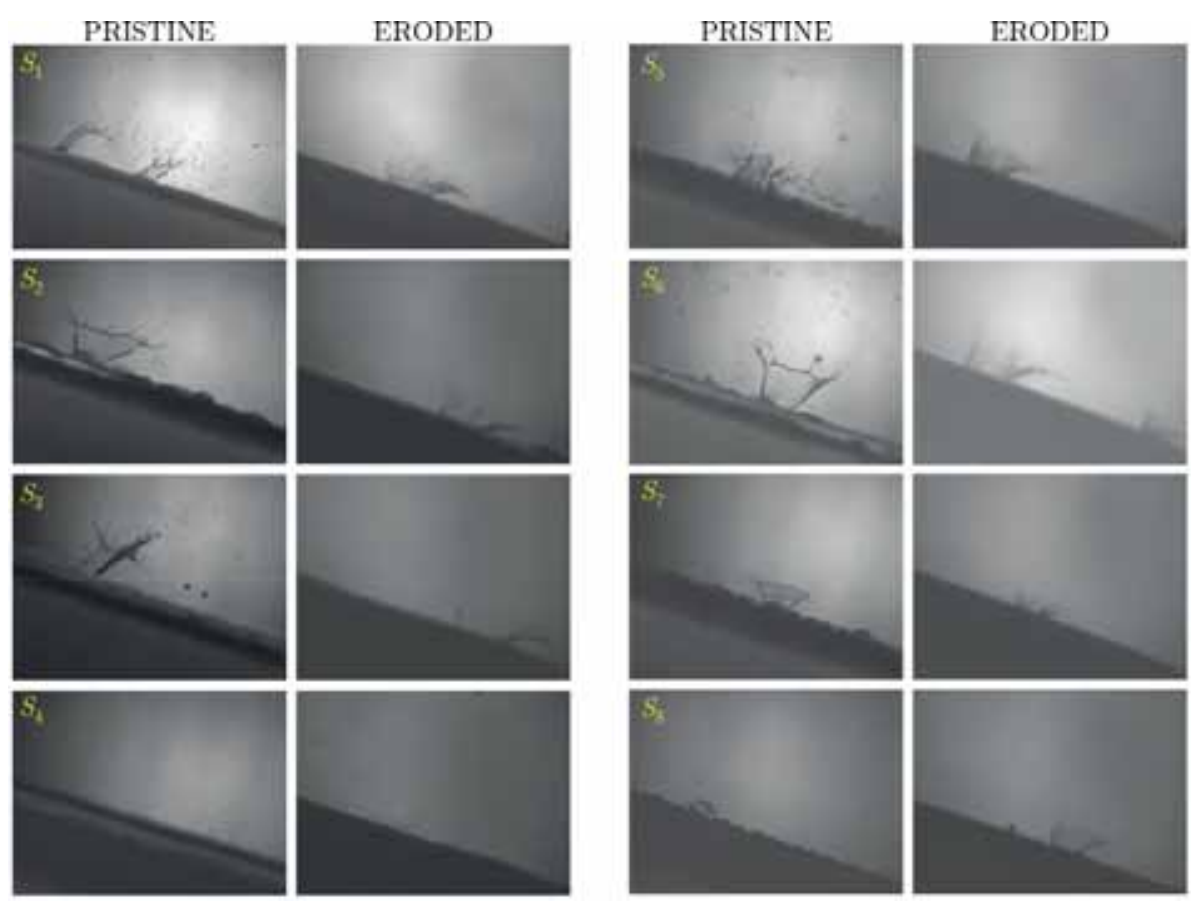

Figure 10: Pre- and post-erosion photographs of splashing structures on specimens $S_{1}-\mathrm{S}_{8}$. The brightness and contrast of the images have digitally been enhanced for visualization purposes. 
(a)

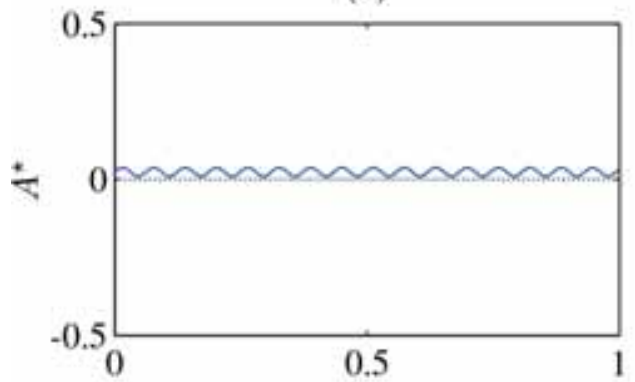

(c)

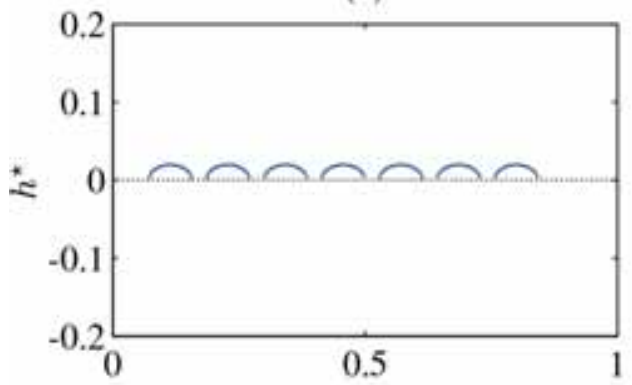

(e)

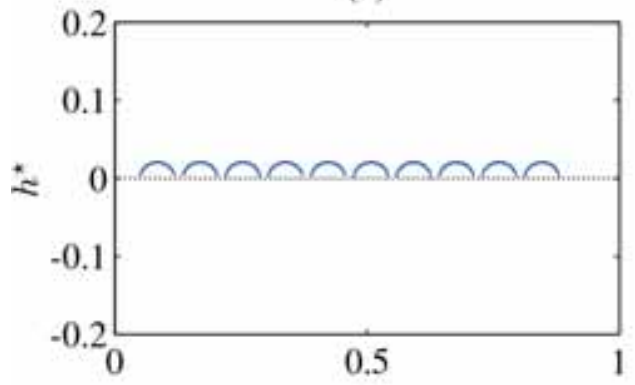

(g)

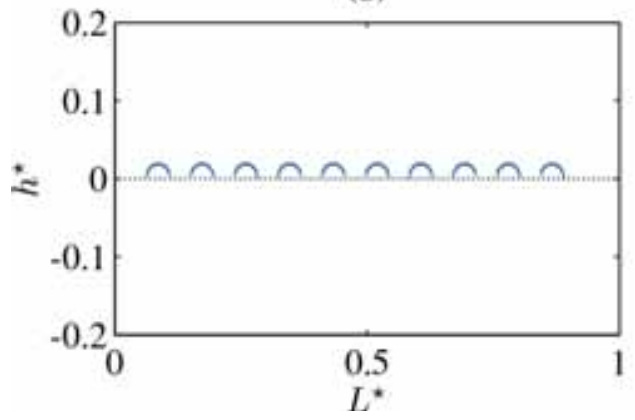

(b)

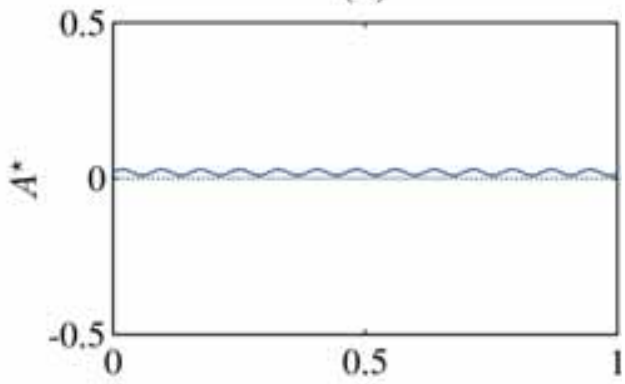

(d)

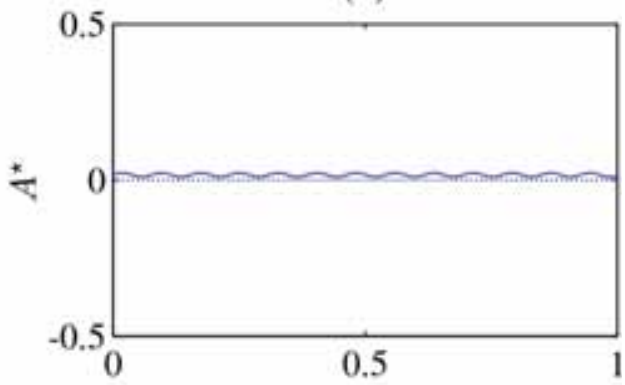

(f)

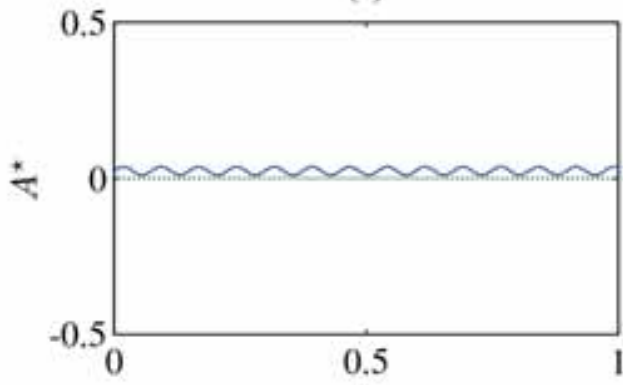

(h)

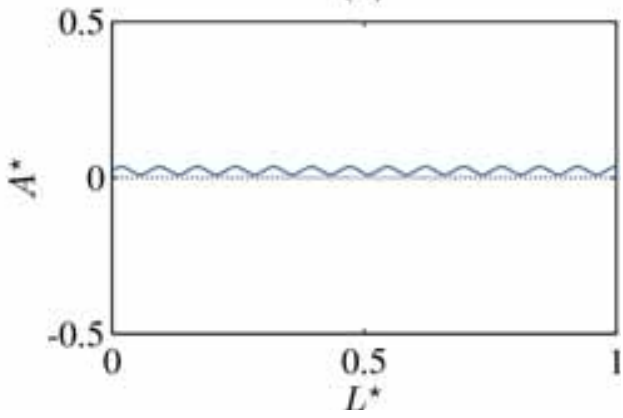

Figure 11: Representative surface structures at $U_{\infty} \approx 35 \mathrm{~m} \cdot \mathrm{s}^{-1}$. The following notations are utilized: (a) $S_{1}^{p}$, (b) $S_{1}^{e}$, (c) $S_{2}^{p}$, (d) $S_{2}^{e}$, (e) $S_{3}^{p}$, (f) $S_{3}^{e}$, (g) $S_{4}^{p}$ and (h) $S_{4}^{e}$. The superscript $p$ and $e$ denote the pristine and eroded states of each specimen. 
(a)

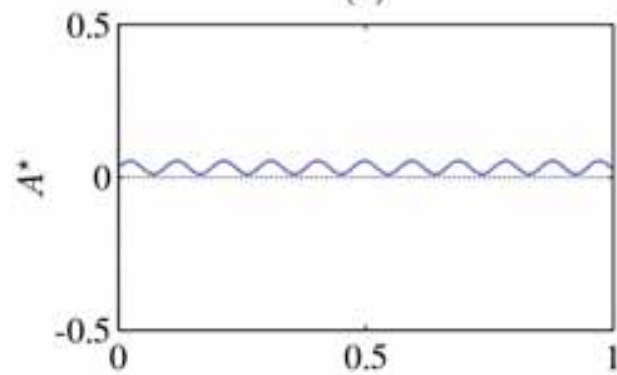

(c)

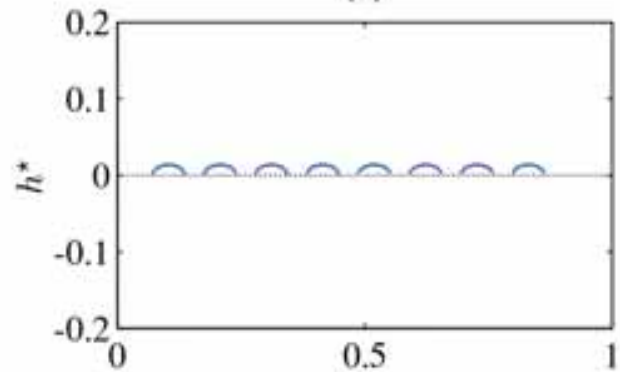

(e)

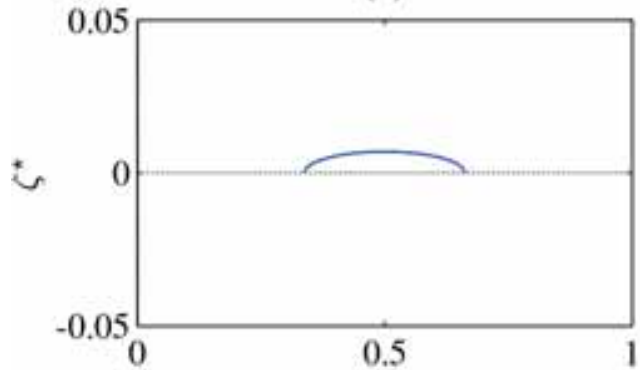

(g)

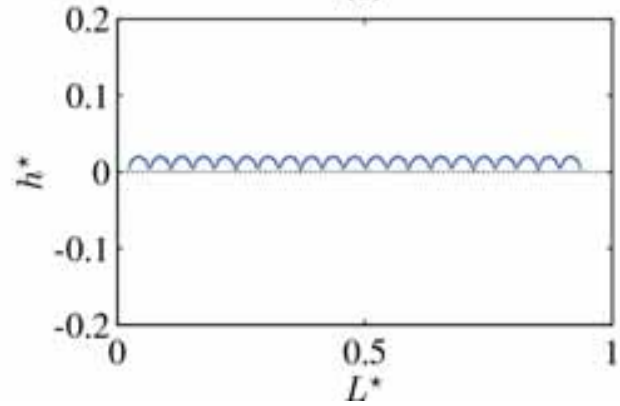

(b)

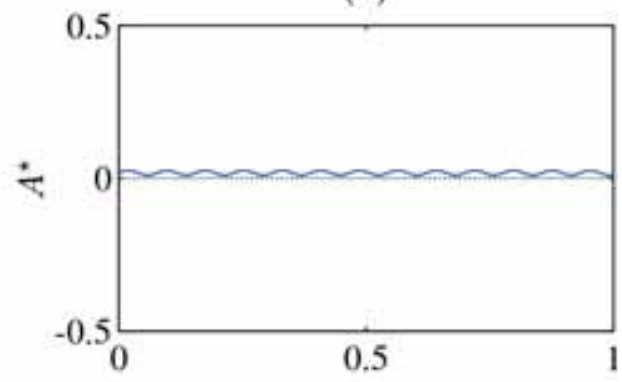

(d)

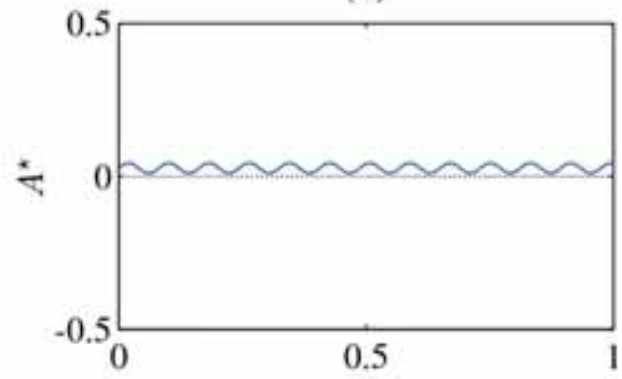

(f)

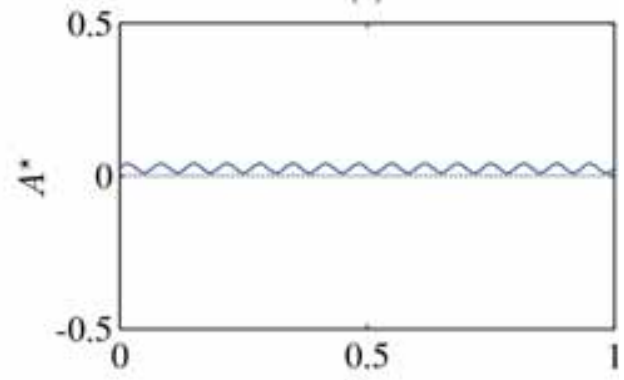

(h)

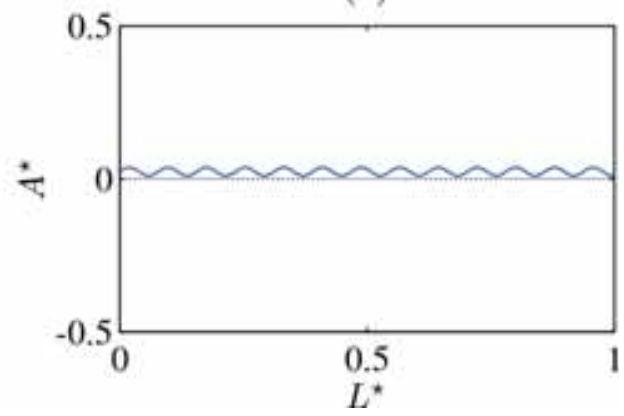

Figure 12: Representative surface structures at $U_{\infty} \approx 35 \mathrm{~m} \cdot \mathrm{s}^{-1}$. The following notations are utilized: (a) $S_{5}^{p}$, (b) $S_{5}^{e}$, (c) $S_{6}^{p}$, (d) $S_{6}^{e}$, (e) $S_{7}^{p}$, (f) $S_{7}^{e}$, (g) $S_{8}^{p}$ and (h) $S_{8}^{e}$. The superscript $\mathrm{p}$ and e denote the pristine and eroded states of the each specimen. 
(a)

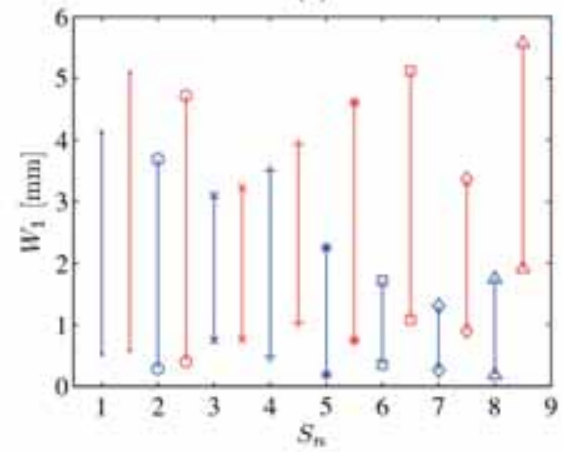

(c)

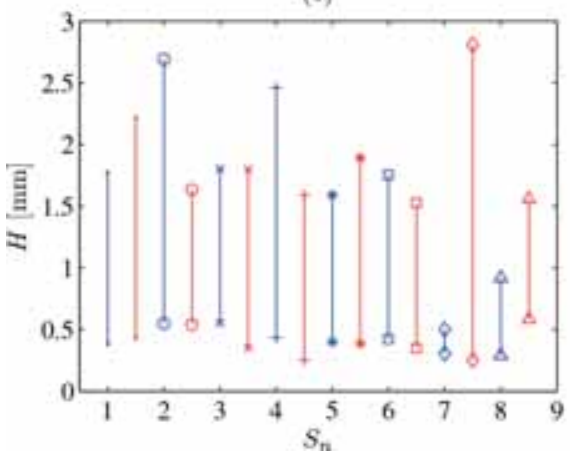

(b)

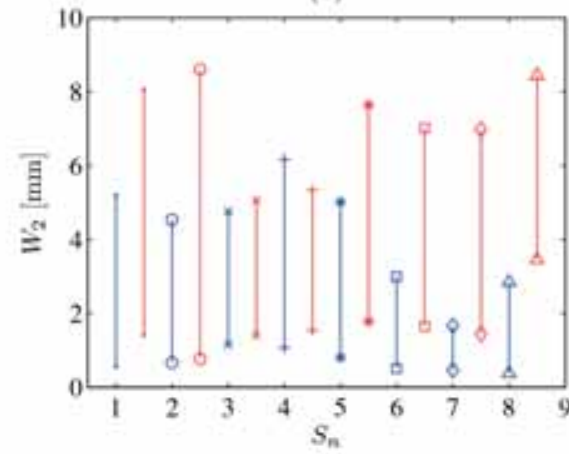

(d)

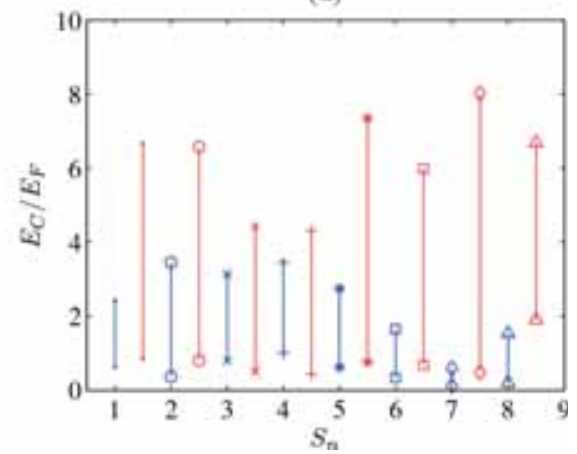

Figure 13: The width at base $W_{1}(\mathrm{a})$, width at top $W_{2}(\mathrm{~b})$, height of corona $H$ (c) and the ratio of the entropy of the corona in relation to the entire entropy of the frame (d) for specimens $S_{n}$, with $n=1,2, \ldots, 8$. The blue color refers to the pristine state and the red color to the eroded state of each specimen. The following symbols are utilized for each specimen: $S_{1}(\cdot), S_{2}(\mathrm{o}), S_{3}(\times), S_{4}(+), S_{5}(*), S_{6}(\square), \mathrm{S} 7(\diamond)$, and $\operatorname{Sg}(\Delta)$.

notable that the height of the corona is reduced for the eroded state of the specimen in comparison to the pristine state, for specimens reinforced with CNTs $S_{2}, \mathrm{~S}_{4}$ and $S_{6}$, which indicates that the hydrophobic nature of these specimens is reduced.

This conjecture is indeed confirmed upon behavior of the fluid structures on the surface for these specimens in Figs. 11 and 12, where erosion of the surface implies that a water film is obtained in contrast to the discrete number of beads formed on the surface in its virgin state.

Due to the large number of parameters obtained, it is more fruitful to combine the parameters to infer general trends about their behavior. Figure 15 shows the behavior of the corona structure based on its skewness, expansion, aspect ratio and area. It can be deduced that the majority of the specimens are skewed to the left in the pristine and eroded states respectively, apart from $S_{6}$ which is skewed to the right prior to erosion. The plurality of the formed corona structures further expand in the eroded state.

The aspect ratio of the corona structures are further more rectangular in shape than square. It is also notable that the two-dimensional area of the corona structure occupies a larger area in the eroded state, in comparison to the virgin state of the material. This may be a consequence of the continuous water film that is evident on most specimens in the aftermath of 
(a)

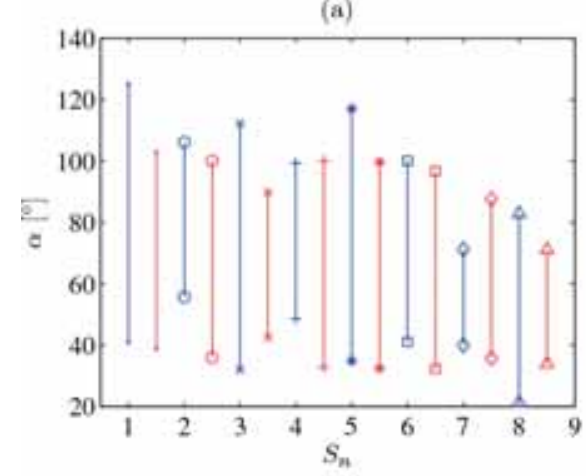

(c)

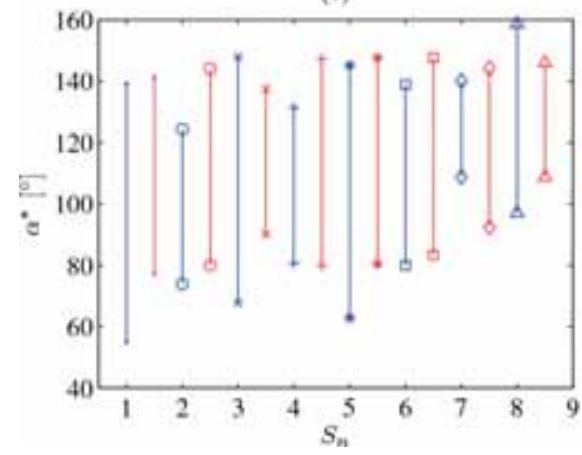

(b)

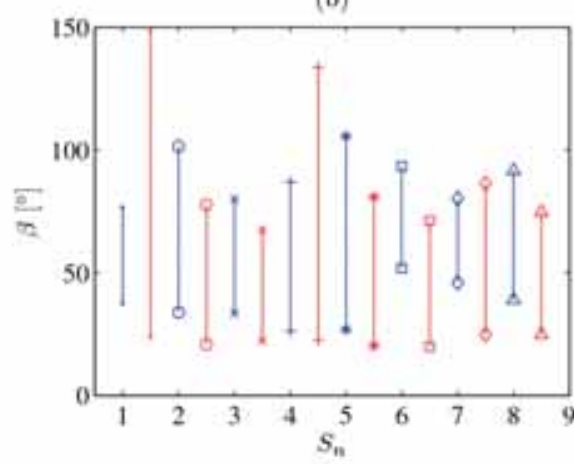

(d)

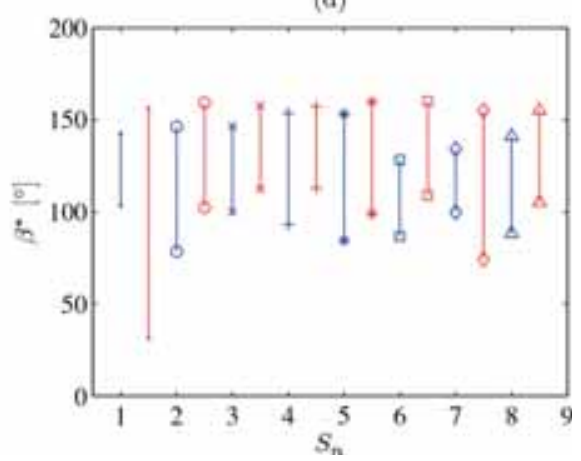

Figure 14: The angle $\alpha(\mathrm{a})$, angle $\beta$ (b), $\alpha^{*}$ (c) and $\beta^{*}$ (d) for specimens $S_{n}$, with $n=1,2$, $\ldots, 8$. The blue color refers to the pristine state and the red color to the eroded state of each specimen. The following symbols are utilized for each specimen: $S_{1}(\cdot), S_{2}(\mathrm{o}), S_{3}(\times), S_{4}(+), S_{5}(*), S_{6}(\square), \mathrm{S} 7(\diamond)$, and $\mathrm{Sg}(\Delta)$.

erosion, allowing for more fluid to become ejected from the surface upon impact. The DSWSAM methodology is also able to output dimensional parameters for the different fluid characteristics. For the considered materials the averaged width and height of the beads with the corresponding standard deviation is given by $\bar{w}=996.59 \pm 298.46$ and $\bar{h}=298.12 \pm$ $44.05 \mu \mathrm{m}$, respectively. The rivulets have an average width and height of $\bar{\xi}=5300.44$ and $\bar{\xi}=$ $113.38 \mu \mathrm{m}$. For the water film the average wavelength and corresponding standard deviation is given by $\bar{\lambda}=1216.34 \pm 135.35 \mu \mathrm{m}$. The averaged amplitude of the water film and corresponding standard deviation is furthermore given by $\bar{A}=204.344 \pm 74.93 \mu \mathrm{m}$.

Given the fact that the DSWSAM represents various fluid structures on a specimen surface by idealized representations, it can be established that there are deviations in shape and size between the encountered structures in comparison to the idealized representations. Despite the encountered differences however, the idealized structure are able to capture the hydrophobic and hydrophilic nature of the specimens. The distance between the beads in particular, serves as an appropriate measure for establishing the extent of hydrophobicity of a surface. A large number of beads, placed adjacent to each other hence corresponds to a highly hydrophobic surface as shown in Fig. 12(g) for the $S_{8}$ specimen. An example of a less hydrophobic surface is depicted in Fig. 11(c) for $S_{2}$. A hydrophilic surface is exemplified by Fig. 12(a) 
(a)

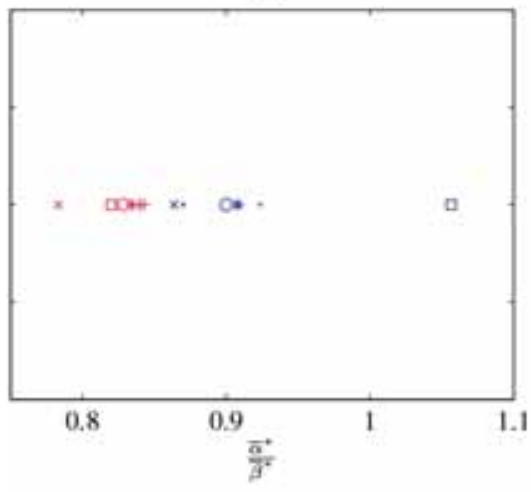

(c)

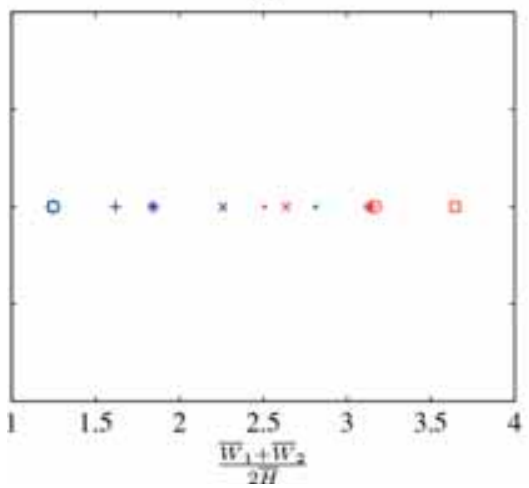

(b)

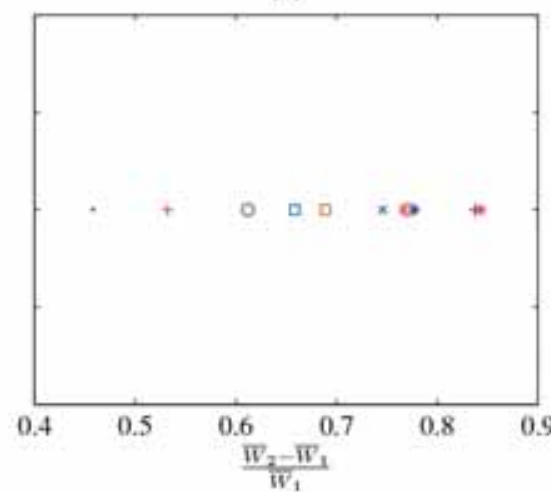

(d)

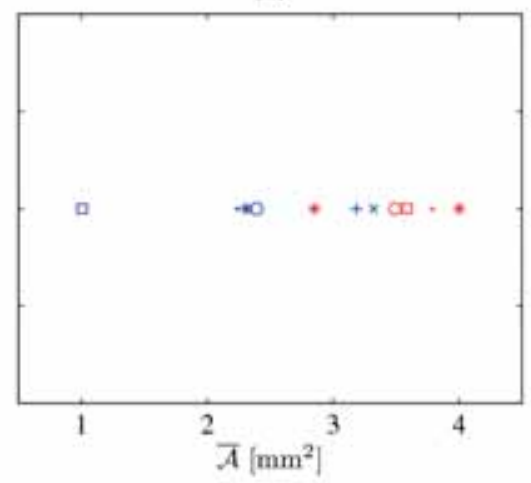

Figure 15: The skewness (a), expansion (b), aspect-ratio (c) and area of the corona (d) for specimens $S_{n}$, with $n=1,2, \ldots, 8$. The blue color refers to the pristine state and the red color to the eroded state of each specimen. The following symbols are utilized for each specimen: $S_{1}(\cdot), S_{2}(0), S_{3}(\times), S_{4}(+), S_{5}(*), S_{6}(\square), \mathrm{S} 7(\diamond)$, and $\mathrm{Sg}(\Delta)$.

for $S_{5}$. The employed methodology is in particular useful for visualizing the differences between the ensuing water films upon erosion of the specimens. The different averaged amplitudes $\bar{A}_{n}$ and water film wave lengths $\bar{\lambda}_{n}$ reduce the number of descriptive parameters of the water to only two. Despite the nominally similar characters of the water films, in particular for the eroded state of the specimens, the visualization tool of the employed methodology is able to discriminate between different progressing idealized waves in accordance with Figs. 11 and 12.

In consideration of the presented parameters in Figs. 13-15, the range of different impact angles has to be considered. Despite the fact that the flow within the Vertical Droplet Tunnel allows for no aerodynamic breakup of the droplet prior to impact, it can be expected that the impact angle $a \neq 70^{\circ}$ in certain instances, which in part can describe the range of values obtained for the presented parameters. Another important factor in this context is the simple fact that the empirical photographs are acquired at different spatial and temporal evolutions of the corona structures following the droplet impact.

For a free stream velocity of $U_{\infty} \approx 35 \mathrm{~m} \cdot \mathrm{s}^{-1}$, the Reynolds number $R e_{d}=12282$, the Weber number $W e_{d} \approx 6725$, the Ohnsorge number $O h=0.0067$, and the Capillary number $C a=0.55$. 
Table 2: The boundary-layer thickness, displacement thickness, momentum thickness, energy thickness, wall shearstress and skin friction for different free stream velocities. All parameters are estimated for $x=L$, where $L$ is the length of the specimen.

\begin{tabular}{lcccccc}
\hline$U_{\infty}\left[\mathrm{m} \cdot \mathrm{s}^{-1}\right]$ & $\delta_{99}[\mu \mathrm{m}]$ & $\delta_{1}[\mu \mathrm{m}]$ & $\delta_{2}[\mu \mathrm{m}]$ & $\delta_{3}[\mu \mathrm{m}]$ & $\tau_{w}\left[\mathrm{~N} \cdot \mathrm{m}^{-2}\right]$ & $c_{f}$ \\
\hline 35 & 463 & 157 & 60.2 & 92.6 & 6.87 & 0.0093 \\
50 & 387 & 132 & 50.3 & 77.5 & 11.7 & 0.0078 \\
60 & 354 & 120 & 46.0 & 70.7 & 15.3 & 0.0071 \\
\hline
\end{tabular}

Table 2, shows the values of the boundary-layer thickness $\delta_{99}$, displacement thickness $\delta_{1}$, momentum thickness $\delta_{2}$, and the energy thickness $\delta_{3}$, wall shear stress $\tau_{w}$, and skin-friction coefficient $c_{f}$ for different free stream velocities.

From Table 2 it is readily established that the approximate size of the boundary layer for a free stream velocity of $35 \mathrm{~m} \cdot \mathrm{s}^{-1}$ is approximately $463 \mu \mathrm{m}$. Therefore, a different structure behavior can be expected at the interface between the boundary layer flow and the outer flow.

The impact of a droplet onto a surface, at high velocities ensues in the growth of fingers and their consequent breakup [18]. The growth of fingers around the periphery of the droplet during spreading is according to Allen [19], a result of the Rayleigh-Taylor Instability, which arises upon acceleration of the interface of fluids with differing densities. The Rayleigh-Taylor instability has in particular empirically been studied by utilizing a paramagnetic fluid combination by Gohardani [20] and Gohardani et al. [21-23]. According to Aziz and Chandra [18], for high enough values resulting in splashing, the maximum spread factor $\widetilde{\xi}_{\max }=0.5 R e^{\frac{1}{4}}$ and the number of fingers $N$, is given by $N=K /(4 \sqrt{3})$, with $K=\left\{W e_{d}\left(R e_{d}\right)^{\frac{1}{2}}\right\}^{\frac{1}{2}}$. Using these relations the maximum spread factor $\tilde{\xi}_{\max } \approx 5.26$ and $N \approx 125$.

\section{CONCLUSIONS}

The DSWSAM methodology is capable of capturing the qualitative observations of the wetting character of different surfaces adequately and express these features quantitatively. The usefulness of this developed methodology is evident upon comparison of nominally similar wetting characteristics of different materials. In particular, in this study the methodology has fully captured the wetting behavior of polymeric matrix composites reinforced with CNTs exhibiting a hydrophobic wetting character prior to erosion and a hydrophilic wetting character, post erosion. The potential role of erosion on the wettability of hydrophobic surfaces is hence highlighted in this study. The change of surface character from a hydrophobic to a hydrophilic, can be of great importance in design aspects related to icephobic surfaces with applications within the aerospace industry. The developed methodology can further be adapted to incorporate more complex features, by addition of advanced modules.

\section{ACKNOWLEDGMENT}

The authors greatly acknowledge the financial support from the European Union for conducting this research as a part of multi-functional layers for safer aircraft composite structures (LAYSA) project, under the Seventh Framework Programme (FP7), including aeronautics. 


\section{REFERENCES}

[1] Shalin, R.E., Polymer Matrix Composites. Springer, p. 440, 1995. ISBN 0412613301. doi: http://dx.doi.org/10.1007/978-94-011-0515-6

[2] Gohardani, O., The influence of erosion and wear on the accretion and adhesion of ice for nano reinforced polymeric composites used in aeronautics. Ph.D. Thesis, Cranfield University, United Kingdom, 2011.

[3] Gohardani, O., Impact of erosion testing aspects on current and future flight conditions. Progress in Aerospace Sciences, 47(4), Elsevier, pp. 280-303, 2011. doi:10.1016/ j.paerosci.2011.04.001.

[4] Gohardani, O., Williamson, D.M. \& Hammond, D.W., Multiple liquid impacts on polymeric matrix composites reinforced with carbon nanotubes. Wear, 294-295, pp. 336-346, 2012. doi:10.1016/j.wear.2012.07.007. doi: http://dx.doi.org/10.1016/j. wear.2012.07.007

[5] Gohardani O. \& Hammond, D.W., Droplet interaction and dynamic wettability of advanced materials used in aeronautics. Presented at the 6th Subrata Chakrabarti International Conference on Fluid Structure Interaction, Fluid Structure Interaction May 9-11, 2011, Orlando, Florida, U.S.A.

[6] Gohardani, A.S., Doulgeris, G. \& Singh, R., Challenges of future aircraft propulsion: a review of distributed propulsion technology and its potential application for the all electric commercial aircraft. Progress in Aerospace Sciences, 47(5), Elsevier, pp. 369-391, 2011. doi:10.1016/j.paerosci.2010.09.001. doi: http://dx.doi.org/10.1016/ j.paerosci.2010.09.001

[7] Gohardani, A.S., A synergistic glance at the prospects of distributed propulsion technology and the electric aircraft concept for future unmanned air vehicles and commercial/military aviation. Progress in Aerospace Sciences, 2012. doi:10.1016/ j.paerosci.2012.08.001.

[8] Gohardani A.S. \& Gohardani O. Ceramic engine considerations for future aerospace propulsion. Aircraft Engineering and Aerospace Technology, 84(2), pp. 75-86, 2012. doi:10.1108/00022661211207884. doi: http://dx.doi.org/10.1108/00022661211207884

[9] Gohardani, O. \& Hammond, D.W., Droplet interaction and dynamic wettability of advanced materials used in aeronautics. Advances in Fluid Mechanics IX, WIT Transactions on Engineering Sciences. ISBN 978-1-84564-600-4, pp. 175-185, 2012.

[10] Hammond, D.W., Luxford, G. \& Ivey P., The Cranfield University Icing Tunnel. In: 41st AIAA Aerospace Sciences Meeting \& Exhibit. 6-9 Jan 2003, Reno, U.S.A.

[11] Young, T., An essay on the cohesion of fluids. Philosophical Transactions of The Royal Society London, 95, pp. 65-87, 1805. doi: http://dx.doi.org/10.1098/rstl.1805.0005

[12] Tadmor, R., Line energy and the relation between advancing, receding, and young contact angles. Langmuir, 20(18), pp. 7659 -7664, 2004. doi: http://dx.doi.org/10.1021/ $\underline{\text { la049410h }}$

[13] Ide, R.F., Comparison of Liquid Water Content measurement techniques in an icing wind tunnel. NASA/TM-1999-209643, 1999.

[14] Rein, M., Phenomena of liquid drop impact on solid and liquid surfaces. Fluid Dynamics Research, 12(2), pp. 61-93, 1993. doi: http://dx.doi.org/10.1016/0169-5983(93)90106-K

[15] Gonzalez, R.C., Woods, R.E. \& Eddins, S.L., Digital Image Processing Using MATLAB, 2nd edn., Gatesmark Publishing, 2009. ISBN 9780982085400.

[16] Schlichting, H. \& Gersten, K., Boundary-Layer Theory. Springer, 2000. ISBN 3-54066270-7. 
[17] Pasandideh-Fard, M., Qiao, Y.M., Chandra, S. \& Mostaghimi, J., Capillary effects during droplet impact on a solid surface. Physics of Fluids, 8(3), pp. 650-659, 1996. doi: http://dx.doi.org/10.1063/1.868850

[18] Aziz, S.D. \& Chandra, S., Impact, recoil and splashing of molten metal droplets. International Journal of Heat Mass Transfer, 43, pp. 2841-2857, 2000. doi: http://dx.doi. org/10.1016/S0017-9310(99)00350-6

[19] Allen, R.F. The role of surface tension in splashing. Journal of Colloid and Interface Science, 51, pp. 350-351, 1975. doi: http://dx.doi.org/10.1016/0021-9797(75)90126-5

[20] Gohardani, O., Experimental investigation of Rayleigh-Taylor instability using a paramagnetic liquid combination. M.Sc. Thesis, University of Arizona, U.S.A., 2008.

[21] Gohardani, O., Oemke, R. \& Jacobs, J.W., PLIF flow visualization of magnetically stabilized Rayleigh-Taylor instability. American Physical Society, 59th Annual Meeting of the APS Division of Fluid Dynamics, November 19-21, 2006, Tampa Bay, Florida, U.S.A.

[22] Gohardani, O., Oemke, R. \& Jacobs, J.W., Experimental study of Rayleigh-Taylor Instability utilizing a paramagnetic liquid combination. American Physical Society, 60th Annual Meeting of the APS Division of Fluid Dynamics, November 18-20, 2007. Salt Lake City, Utah, U.S.A.

[23] Gohardani, O. \& Jacobs, J.W. Experimental investigation of the Rayleigh-Taylor Instability using a paramagnetic liquid combination. Proceedings of the 11th International Workshop on the Physics of Compressible Turbulent Mixing (IWPCTM), July 13-18, 2008, Santa Fe, New Mexico, U.S.A.

[24] Gohardani, O., The Exploration of Icephobic Materials and Their Future Prospects in Aircraft Icing Applications. J Aeronaut Aerospace Eng, 1, p. e116, 2012. doi:10.4172/ 2168-9792.1000e116. 